\title{
PSEUDOHOLOMORPHICITY OF CLOSED MINIMAL SURFACES IN CONSTANTLY CURVED 4-SPACES
}

\author{
CHI-MING YAU
}

(Communicated by Jonathan M. Rosenberg)

\begin{abstract}
The twistor lifts of a minimal surface in a constantly curved 4-space are studied. Computing the Laplacian of the length of their differentials provides a topological condition for pseudoholomorphicity. Geometric conditions for holomorphicity of surfaces in flat spaces are found.
\end{abstract}

The pseudoholomorphicity condition for minimal surfaces has been studied by various authors, e.g., Calabi [2], Chern [3], Eells and Wood [6]. For a long time, it has been known that the condition is fulfilled a priori when the surface is of genus zero and the ambient space is constantly curved. One would thus like to study the higher genus cases. Using the method of twistor lifts, and an analog of the diagonalization of the second fundamental form, Lemma 1.1, we obtain the

Theorem. Let $M$ be a closed minimal surface in a four-dimensional space of positive constant curvature $k$. Then $M$ is pseudoholomorphic if $\left|\chi\left(T M^{\perp}\right)\right|>$ $-2 \chi(M)$, where $\chi(M)$ and $\chi\left(T M^{\perp}\right)$ are the Euler characteristics of $M$ and its normal bundle $T M^{\perp}$ respectively. In this case, the immersion is determined up to an isometry of $N$ by the curvature function of $M$. Furthermore, if $k=$ 1 , then the area of $M$ is an integral multiple of $2 \pi$.

Note. R. Bryant [1] and Chern and Wolfson [5] showed that closed pseudoholomorphic minimal surfaces of arbitrary genus exist in $S^{4}$; they called them superminimal.

\section{THE SECOND FUNDAMENTAL FORM}

Let $\phi: M^{2} \rightarrow N^{2+p}$ be an immersion of a surface into a Riemannian manifold. We denote the tangent bundles of $M$ and $N$ by $T M$ and $T N$ respectively, and the dual of an adapted orthonormal frame field $\left\{e_{1}, \ldots, e_{2+p}\right\}$ by $\left\{\omega_{1}, \ldots, \omega_{2+p}\right\}$. From now on, unless otherwise noted, we will adhere to the

Received by the editors April 24, 1989 and, in revised form, December 14, 1989.

1980 Mathematics Subject Classification (1985 Revision). Primary 53C42, 58E20.

Key words and phrases. Minimal surfaces, constantly curved 4-spaces, twistor lifts, pseudoholomorphicity. 
following convention on the range of indices.

$$
\begin{aligned}
& 1 \leq A, B, C, \ldots \leq 2+p \\
& 1 \leq i, j, \ldots \leq 2 \\
& 3 \leq \alpha, \beta, \ldots \leq 2+p
\end{aligned}
$$

To fix notations, we write the structural equations of $N$ as:

$$
\begin{gathered}
\mathrm{d} \omega_{A}=\sum_{B} \omega_{A B} \wedge \omega_{B}, \quad \omega_{A B}+\omega_{B A}=0, \\
\mathrm{~d} \omega_{A B}=\sum_{C} \omega_{A C} \wedge \omega_{C B}-\frac{1}{2} \sum_{C, D} R_{A B C D} \omega_{C} \wedge \omega_{D},
\end{gathered}
$$

where $\omega_{A B}$ are the connection forms and $R_{A B C D}$ is the curvature tensor of $N$. Similar equations also hold on $M$ and its normal bundle $T M^{\perp}$, whose curvature tensors would be denoted by $K_{i j k l}$ and $K_{\alpha \beta i j}$ respectively. The second fundamental form $\left(h_{i j}^{\alpha}\right)_{1 \leq i, j \leq 2}$ is defined by the equations

$$
\omega_{i \alpha}=\sum_{j} h_{i j}^{\alpha} \omega_{j}, \quad h_{i j}^{\alpha}=h_{j i}^{\alpha}
$$

$\phi: M^{2} \rightarrow N^{2+p}$ is called minimal if $\sum_{i} h_{i i}^{\alpha}=0$ for all $\alpha$ everywhere.

Lemma 1.1. Let $\phi: M^{2} \rightarrow N^{2+p}$ be a minimal immersion $(p \geq 2)$. At every point $x$ in $M$, there exist local adapted orthonormal frame fields $\left\{e_{1}, \ldots, e_{2+p}\right\}$ so that at $x$, the second fundamental form becomes

$$
\begin{gathered}
\left(h_{i j}^{3}\right)_{1 \leq i, j \leq 2}=\left(\begin{array}{ll}
0 & b \\
b & 0
\end{array}\right), \quad\left(h_{i j}^{4}\right)_{1 \leq i, j \leq 2}=\left(\begin{array}{cc}
c & 0 \\
0 & -c
\end{array}\right), \\
\left(h_{i j}^{5}\right)_{1 \leq i, j \leq 2}=\cdots=\left(h_{i j}^{2+p}\right)_{1 \leq i, j \leq 2}=0 .
\end{gathered}
$$

Proof. Consider the map $A: T M^{\perp} \rightarrow T M \otimes T M$ defined by

$$
A\left(e_{\alpha}\right)=\sum_{i, j} h_{i j}^{\alpha} e_{i} \otimes e_{j} .
$$

By minimality of the immersion, the image of $A$ lies in the traceless symmetric part of $T M \otimes T M$ which is of rank two. The rank of $A$ at $x$ hence is at most equal to two. We assume that $A$ is of rank two at $x$, the proof for the other cases are trivial. Choose a basis $\left\{e_{5}, \ldots, e_{2+p}\right\}$ for the kernel of $A$, and so $h_{i j}^{\alpha}=0$ for $\alpha \geq 5$. Next, we choose $\left\{e_{3}, e_{4}\right\}$ in order to diagonalize the bilinear form $\ll \cdot, \cdot \gg$, which is defined as $\ll e_{\alpha}, e_{\beta} \gg=\sum_{i, j} h_{i j}^{\alpha} h_{i j}^{\beta}$. Thus $\sum_{i, j} h_{i j}^{3} h_{i j}^{4}=0$. And we choose $\left\{e_{1}, e_{2}\right\}$ to diagonalize $\left(h_{i j}^{4}\right)_{1 \leq i, j \leq 2}$. We obtain

$$
\left(h_{i j}^{3}\right)_{1 \leq i, j \leq 2}=\left(\begin{array}{cc}
a & b \\
b & -a
\end{array}\right), \quad\left(h_{i j}^{4}\right)_{1 \leq i, j \leq 2}=\left(\begin{array}{cc}
c & 0 \\
0 & -c
\end{array}\right) .
$$


The equation $\sum_{i, j} h_{i j}^{3} h_{i j}^{4}=0$ tells us that $a=0$. Q.E.D.

Assuming that $N$ has constant curvature $k$, we can compute along the lines of Chern, DoCarmo, and Kobayashi [4] with extra help from Lemma 1.1. Denote the Laplacian of the second fundamental form as $\Delta h_{i j}^{\alpha}$ and put $\sigma=\sum_{i, j, \alpha}\left(h_{i j}^{\alpha}\right)^{2}$, then we have

$$
-\frac{1}{2} \sum_{i, j, \alpha} h_{i j}^{\alpha} \Delta h_{i j}^{\alpha}=\frac{3}{4} \sigma^{2}-k \sigma-\left(b^{2}-c^{2}\right)^{2} .
$$

Integrating both sides of the above identity, we obtain a proof of

Proposition 1.2. If $\sigma \leq 4 / 3$ everywhere on a closed minimal surface $M$ in $S^{2+p}$, then either $M$ is totally geodesic, or $\sigma=4 / 3$ everywhere.

Proposition 1.3. Let $M$ be a closed minimal surface in a 4-space of constant curvature $k$, then

$$
\frac{1}{2} \int\left(K^{\perp}\right)^{2}+\int K^{2} \geq 2 \pi k \chi(M),
$$

where $K=K_{1212}, K^{\perp}=K_{3412}$ are the curvatures of $M$ and $T M^{\perp}$ respectively. Proof. The identity (1.6) can be rewritten as

$$
-\frac{1}{2} \sum_{i, j, \alpha} h_{i j}^{\alpha} \Delta h_{i j}^{\alpha}=\left(K^{\perp}\right)^{2}-2 K(k-K) .
$$

The proof is completed by integration. Q.E.D.

\section{PSEUDOHOLOMORPHICITY}

Note. From now on, unless otherwise stated, we assume that $N$ is of dimension 4 , oriented, constantly curved, and $\phi: M^{2} \rightarrow N^{4}$ is a minimal immersion.

The involutive $*$ operator on $\wedge^{2} T N$ is defined by $\alpha \wedge(* \beta)=\langle\alpha, \beta\rangle$ $e_{1} \wedge \cdots \wedge e_{4}$. Decompose $\Lambda^{2} T N$ into the \pm -eigenspaces of $*: \Lambda^{2} T N=$ $\bigwedge_{+}^{2} T N \oplus \Lambda_{-}^{2} T N$. The Gauss lift of $\phi: M^{2} \rightarrow N^{4}$ is $G=e_{3} \wedge e_{4}$, and the twistor lifts are $G^{ \pm}=\left(e_{1} \wedge e_{2} \pm e_{3} \wedge e_{4}\right) / \sqrt{2}$.

Choose $\left\{e_{1}, \ldots, e_{4}\right\}$ as in Lemma 1.1, then by equation (1.1),

$$
\left\|\mathrm{d} G^{ \pm}\right\|^{2}=\left\|\omega_{23} \pm \omega_{14}\right\|^{2}+\left\|\omega_{24} \pm \omega_{31}\right\|^{2}=2(b \pm c)^{2} .
$$

Hence, $\quad\left\|\mathrm{d} G^{+}\right\|^{2}\left\|\mathrm{~d} G^{-}\right\|^{2}=4\left(b^{2}-c^{2}\right)^{2}$.

As in Chern [3], consider the antiholomorphic quartic differential: $\tau=$ $\sum_{\alpha} H_{\alpha}^{2} \bar{\zeta}^{4}$ where $H_{\alpha}=h_{11}^{\alpha}-h_{22}^{\alpha}+2 i h_{12}^{\alpha}$ and $\zeta=\omega_{1}+i \omega_{2}$. By Lemma $1.1 H_{3}=2 i b, H_{4}=2 c$, and $\tau=\left(-4 b^{2}+4 c^{2}\right) \bar{\zeta}^{4}$. Therefore,

$$
\|\tau\|^{2}=4^{2} 2^{4}\left(b^{2}-c^{2}\right)^{2}=64\left\|\mathrm{~d} G^{+}\right\|^{2}\left\|\mathrm{~d} G^{-}\right\|^{2} .
$$

Definition 2.1. $\phi: M^{2} \rightarrow N^{4}$ is called pseudoholomorphic if $\tau \equiv 0$. 
Proposition 2.2. If $\phi: M^{2} \rightarrow N^{4}$ is not pseudoholomorphic, then $\left\|\mathrm{d} G^{ \pm}\right\|^{2}$ has only isolated zeros of finite order, and

$$
\frac{1}{4} \triangle \log \left\|\mathrm{d} G^{ \pm}\right\|^{2}=K \pm \frac{1}{2} K^{\perp} .
$$

Proof. As $\tau$ is antiholomorphic, $\|\tau\|^{2}$ has only isolated zeros of finite order if it is nonvanishing, and so is $\left\|\mathrm{d} G^{ \pm}\right\|^{2}$ by equation (2.2).

Extend the Riemannian metric $<\cdot, \cdot\rangle$ to the complexified normal bundle by complex bilinearity. Consider $\xi=<\sum_{\alpha} H_{\alpha} e_{\alpha}, u>\bar{u} \otimes \bar{\zeta}^{2}$ where $u=e_{3}+i e_{4}$. As in Chern [3],

$$
\mathrm{d} H_{\alpha}+\sum_{\beta} H_{\beta} \omega_{\beta \alpha}+2 i \omega_{12} H_{\alpha} \equiv 0 \quad(\bmod \zeta),
$$

and $\nabla u=-i \omega_{34} u$, hence $\nabla \xi \equiv 0(\bmod \zeta)$, i.e., $\xi$ is antiholomorphic. Therefore,

$$
\frac{1}{4} \Delta \log \|\xi\|^{2}=K+\frac{1}{2} K^{\perp} .
$$

On the other hand, by Lemma $1.1, \xi=2 i(b+c) u \otimes \bar{\zeta}^{2}$, hence $\|\xi\|^{2}=$ $32(b+c)^{2}=16\left\|\mathrm{~d} G^{+}\right\|^{2}$. Redefining $\xi$ as $<\sum_{\alpha} H_{\alpha} e_{\alpha}, \bar{u}>u \otimes \bar{\zeta}^{2}$, gives $\frac{1}{4} \Delta \log \left\|\mathrm{d} G^{-}\right\|^{2}=K-\frac{1}{2} K^{\perp}$. Q.E.D.

Proof of the theorem. Assume that $M$ is not pseudoholomorphic, thus neither $\left\|\mathrm{d} G^{ \pm}\right\|^{2}$ is identically zero. Let $n_{ \pm}$be the total numbers of zeros, counting multiplicities, of $\left\|\mathrm{d} G^{ \pm}\right\|^{2}$. Integrating both sides of equation (2.3), the GaussBonnet and Residue formula gives

$$
\chi(M) \pm \frac{1}{2} \chi\left(T M^{\perp}\right)=-n_{ \pm} / 2 \leq 0 .
$$

This implies that $\left|\chi\left(T M^{\perp}\right)\right| \leq-2 \chi(M)$.

That the immersion is determined up to an isometry of $N$ by the curvature function of $M$ when $M$ is pseudoholomorphic is a result of Chern [3].

By using the elementary identity $(b \pm c)^{2}=b^{2} \pm 2 b c+c^{2}$ and the Gauss equation, the following can be proved from equation (2.1) when $M$ is minimal.

$$
\frac{1}{2}\left\|\mathrm{~d} G^{ \pm}\right\|^{2}=k-K \mp K^{\perp}
$$

If $M$ is pseudoholomorphic then one of $\left\|\mathrm{d} G^{ \pm}\right\|^{2}$ is identically zero. Suppose $\left\|\mathrm{d} G^{+}\right\|^{2}$ is zero. Then integrating equation (2.7) over $M$ gives

$$
k \operatorname{Area}(M)=2 \pi\left(\chi(M)+\chi\left(T M^{\perp}\right)\right) .
$$

The area of $M$ is thus an integral multiple of $2 \pi$ when $k=1$. Q.E.D.

We denote the four-dimensional Euclidean space and the four-torus with the flat canonical metrics by $E^{4}$ and $T^{4}$ respectively. 
Proposition 2.3. For a closed minimal surface $M$ in a four-dimensional space of nonpositive constant curvature, $\left|\chi\left(T M^{\perp}\right)\right| \leq-\chi(M)$.

Proof. From equation (2.7), $-K \mp K^{\perp}=\frac{1}{2}\left\|\mathrm{~d} G^{ \pm}\right\|^{2}-k \geq 0$. Apply the GaussBonnet. Q.E.D.

Lemma 2.4. An immersed surface in $E^{4}$ or $T^{4}$ is \pm -holomorphic with respect to some complex structure iff it is pseudoholomorphic.

Proof. By translational invariance, $G^{ \pm}$can be regarded as a mapping into the unit spheres of $\Lambda_{ \pm}^{2} R^{4}$, which in turn can be identified with the space of \pm orientation preserving complex structures in $E^{4}$ or $T^{4}$. Q.E.D.

We have the following local geometric characterization of holomorphic curves in $C^{2}$.

Corollary 2.5. An immersed surface (not neccessarily complete or minimal) in $E^{4}$ is holomorphic with respect to some positively oriented complex structure of $E^{4}$ iff it satisfies the equation

$$
\sigma-2 K^{\perp}=0,
$$

where $\sigma=\sum_{i, j, \alpha}\left(h_{i j}^{\alpha}\right)^{2}$. Similarly, it is holomorphic with respect to some negatively oriented complex structure iff $\sigma+2 K^{\perp}=0$.

Proof. As we are not assuming the surface to be minimal, we do not use Lemma 1.1. Nevertheless, as in equation (2.1), $\left\|\mathrm{d} G^{ \pm}\right\|^{2}=\left\|\omega_{23} \pm \omega_{14}\right\|^{2}+\left\|\omega_{24} \pm \omega_{31}\right\|^{2}$. Expanding it by equation (1.3) gives $\left\|\mathrm{d} G^{ \pm}\right\|^{2}=\sigma \mp 2 K^{\perp}$. Thus $G^{ \pm}$is constant iff $\sigma \mp 2 K^{\perp}=0$. Q.E.D.

We can extend a result of Xavier [8] as:

Corollary 2.6. A complete minimal surface in $E^{4}$ is \pm -holomorphic with respect to some complex structure if one of the images of $G^{ \pm}$misses 7 points.

Proof. Using the same proof as Xavier's theorem, it is easy to see that $G^{ \pm}$is constant if it misses 7 points. Q.E.D.

Combining the proof of Proposition 2.3 and Lemma 2.4, we obtain:

Corollary 2.7. A closed minimal surface in $T^{4}$ is \pm -holomorphic with respect to some complex structure iff $\left|\chi\left(T M^{\perp}\right)\right|=-\chi(M)$.

\section{REFERENCES}

1. R. L. Bryant, Conformal and minimal immersions of compact surfaces into the 4-sphere, J. Differential Geom. 17 (1982), 455-473.

2. E. Calabi, Minimal immersions of surfaces in Euclidean spheres, J. Differential Geom. 1 (1967), 111-125.

3. S. S. Chern, On the minimal immersion of the two-sphere in a space of constant curvature, Problems in Analysis, Princeton Univ. Press, 1970, pp. 27-40. 
4. S. S. Chern, M. Do Carmo, and S. Kobayashi, Minimal submanifolds of a sphere with second fundamental form of constant length, Functional Analysis and Related Fields, SpringerVerlag, 1979, pp. 59-75.

5. S. S. Chern and J. C. Wolfson, Minimal surfaces by moving frames, Amer. J. Math. 105 (1983), 59-83.

6. J. Eells and J. C. Wood, Harmonic maps from surfaces to complex projective spaces, Adv. in Math. 49 (1983), 217-263.

7. M. J. Micallef, Stable minimal surfaces in Euclidean space, J. Differential Geom. 19 (1984), 57-84.

8. F. Xavier, The Gauss map of a complete non-flat minimal surface cannot omit 7 points of the sphere, Ann. of Math. 113 (1981), 211-214.

Department of Mathematics, University of Toledo, Toledo, Ohio 43606 